\title{
Capillary Ionization and Jumps of Capacitive Energy Stored in Mesopores
}

\author{
Carolina Cruz, Svyatoslav Kondrat,* Enrique Lomba, and Alina Ciach
}

Cite This: J. Phys. Chem. C 2021, 125, 10243-10249

Read Online

ABSTRACT: We study ionic liquid-solvent mixtures in slit-shaped nanopores wider than a few ion diameters. Using a continuum theory and generic thermodynamic reasoning, we reveal that such systems can undergo a capillary ionization transition. At this transition, the pores spontaneously ionize or deionize upon infinitesimal changes of temperature, slit width, or voltage. Our calculations show that a voltage applied to a pore may induce a capillary ionization, which - counterintuitively - is followed by a re-entrant deionization as the voltage increases. We find that such ionization transitions produce sharp jumps in the accumulated charge and stored energy, which may find useful applications in energy storage and heat-to-energy conversion.

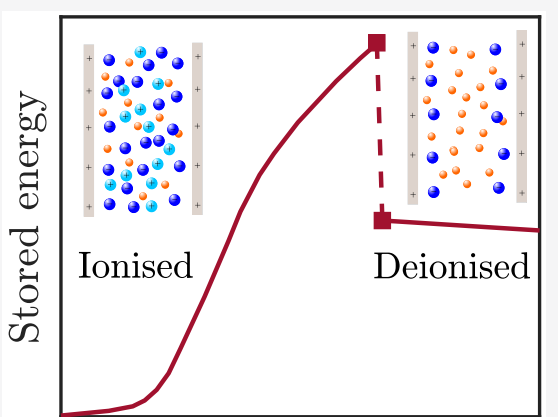

Temperature

\section{INTRODUCTION}

Ionic liquids (ILs) under confinement play a key role in science and technology, exhibiting remarkable properties and finding applications in energy storage, ${ }^{1-4}$ capacitive deionisation, $^{5-7}$ heat-to-energy conversion, ${ }^{8-10}$ etc. For instance, subnanometer pores filled with an electrolyte provide the highest achievable capacitance ${ }^{11-13}$ and stored energy, ${ }^{14}$ though sluggish dynamics. ${ }^{15-18}$ Electrodes with mesoscale pores avoid a typically poor interpore connectivity of microporous electrodes, ${ }^{19}$ enabling faster charging. ${ }^{20,21}$ Using neat ILs, which have large electrochemical windows, ${ }^{22-24}$ enhances the electrical energy stored in micro- and mesopores. ${ }^{25}$ Unfortunately, neat ILs exhibit slow dynamics; mixing ILs with solvents, such as acetonitrile or water, enhances the IL conductivity ${ }^{26-29}$ and speeds up the charging kinetics. $^{30}$

Previous work has focused on micro- and mesopores filled with IL-solvent mixtures far from phase transitions. However, confined fluids show an exciting physics close to state transformations. A classic example is a capillary condensation, ${ }^{31,32}$ which has numerous practical applications, particularly in determining the pore-size distribution of micro- and mesoporous materials. ${ }^{33-35}$ In this article, we study IL-solvent mixtures liable to phase separation, confined in pores substantially wider than the ion diameter. Using a simple continuum theory and general thermodynamic arguments, we demonstrate that the pores can become spontaneously ionized or deionized in response to small changes in temperature or the applied potential difference. We show that such capillary ionization goes along with abrupt changes in charge and energy storage, which may find practical applications in electrochemical energy storage and generation.

\section{MODEL}

We consider a mixture of a room-temperature ionic liquid and solvent confined into a slit mesopore of a supercapacitor's electrode, with the potential difference $U$ applied to the pore walls with respect to the bulk electrolyte (Figure 1). We model solvent implicitly and assume that the system is translational invariant in the latteral $x, y$ directions. This system can be described by the grand potential ${ }^{36,37}$

$$
\begin{aligned}
\beta \Omega\left[\rho_{ \pm}, u\right] / A= & \int_{0}^{w}\left[\rho_{+} \ln \left(a^{3} \rho_{+}\right)+\rho_{-} \ln \left(a^{3} \rho_{-}\right)+\beta \rho f_{\mathrm{ex}}(\rho)\right] \mathrm{d} z \\
& +\int_{0}^{w}\left[\beta e c u-\frac{1}{4 \pi \epsilon_{\mathrm{r}}}\left(\frac{\partial u}{\partial z}\right)^{2}\right] \mathrm{d} z \\
& +\beta K\left\{\int_{0}^{w}\left[\frac{\xi_{0}^{2}}{2}\left(\frac{\partial \rho}{\partial z}\right)^{2}-\frac{1}{2} \rho^{2}\right] \mathrm{d} z\right. \\
& \left.+\sum_{\alpha=\{0, w\}}\left(\frac{\xi_{0}}{2} \rho_{\alpha}^{2}-h_{\mathrm{s}} \rho_{\alpha}\right)\right\}-\beta \mu \int_{0}^{w} \rho \mathrm{d} z
\end{aligned}
$$

Received: February 5, 2021

Revised: April 21, 2021

Published: April 30, 2021 


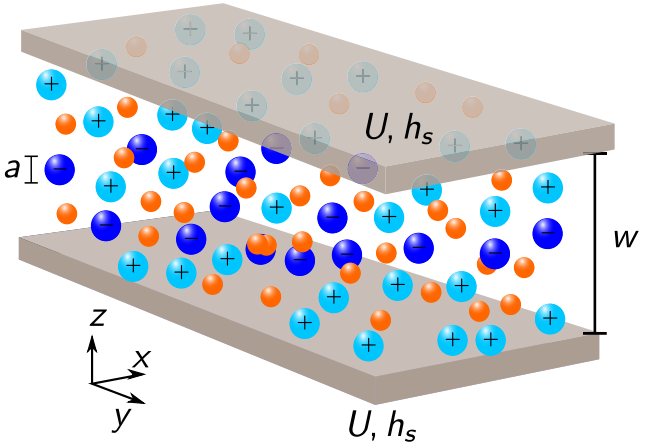

Figure 1. Ionic liquid-solvent mixture in a slit mesopore. The pore walls are separated by a distance $w$. The ion diameter $a$ is the same for cations (light blue spheres) and anions (dark blue spheres). Orange spheres represent solvent molecules, modeled implicitly in eq $1 . h_{\mathrm{s}}$ is the surface field that describes the preference of pore walls for ions or solvent. The potential difference $U$ is applied to the pore walls with respect to the bulk electrolyte (not shown). The system is translational invariant in the lateral $x, y$ directions.

where $\beta=1 /\left(k_{\mathrm{B}} T\right)\left(k_{\mathrm{B}}\right.$ is the Boltzmann constant and $T$ is temperature), $A$ is the surface area, $w$ is the slit width, $\rho_{ \pm}$are the cation and anion densities, and $\mu$ is the chemical potential. The charge density is $c=\rho_{+}-\rho_{-}$(in units of the elementary charge $e$ ) and the total ion density $\rho=\rho_{+}+\rho_{-}$.

The first term in eq 1 is the entropic contribution, which consists of the ideal gas entropy and the free energy density due to excluded volume interactions, $f_{\mathrm{ex}}$, approximated here by the Carnahan-Starling expression,

$$
\beta f_{\mathrm{ex}}(\rho)=\frac{4 \eta-3 \eta^{2}}{(1-\eta)^{2}}-1
$$

where $\eta=\pi a^{3} \rho / 6$ is the ion packing fraction and $a$ is the ion diameter. The second term in eq 1 is the electrostatic energy, where $u$ is the local electrostatic potential and $\epsilon_{\mathrm{r}}$ is the relative dielectric constant. At room temperature, $\epsilon_{\mathrm{r}}$ ranges from 80 for water down to about 10 for less polar solvents like alkohols and increases monotonically with increasing temperature. ${ }^{38-40}$ Correspondingly, the Bjerrum length $\lambda_{\mathrm{B}}=e^{2} /\left(\epsilon_{\mathrm{r}} k_{\mathrm{B}} T\right)$ acquires a relatively weak dependence on temperature and can increase or decrease with temperature. For simlicity, however, we assume here a temperature-independent Bjerrum length $\lambda_{\mathrm{B}}=a$ (corresponding to $\epsilon_{\mathrm{r}} \approx 80$ at room temeprature) and note that the temperature dependence and the choice of $\epsilon_{\mathrm{r}}$ do not affect our results qualitatively (cf. Figure S5).

The third term in eq 1 arises due to van der Waals interactions, with $K$ measuring the strength of the interactions and $\xi_{0}$ their spatial extension. ${ }^{36}$ The parameter $K$ sets a temperature (energy) scale, which we express via the critical temperature of a bulk system, as described below. The parameter $\xi_{0}$ is comparable to the molecular dimension and we set it equal to the ion diameter in all calculations.

Ionophilicity $h_{\mathrm{s}}$ in eq 1 describes the preference of pore walls for ions or solvent. A negative $h_{\mathrm{s}}$ means that the pore walls favor solvent, while $h_{\mathrm{s}}>0$ means that the walls prefer ions, and we assume that this preference is the same for cations and anions. We focus on ionophilic and weakly ionophobic $\left(h_{\mathrm{s}} \approx\right.$ $0)$ pore walls and note that even for an "ionophilic" wall, the ion density at the wall can be lower than in the bulk, provided the bulk ion density $\rho_{b}>h_{s} / \xi_{0}{ }^{36}$

The equilibrium properties of the system are determined by a minimum of $\Omega$. Minimization of $\Omega$ with respect to $\rho_{ \pm}$and $u$ leads to differential equations which we solved numerically (Section S1).

\section{RESULTS AND DISCUSSION}

Bulk System. In a bulk system, that is, outside of the pores, eq 1 simplifies to $\beta \Omega\left(\bar{\rho}_{\mathrm{b}}\right) / A=-\beta K \bar{\rho}_{\mathrm{b}}^{2} / 2+\bar{\rho}_{\mathrm{b}} \ln \left(\bar{\rho}_{\mathrm{b}} / 2\right)+$ $\beta \bar{\rho}_{\mathrm{b}} f_{\mathrm{ex}}\left(\bar{\rho}_{b}\right)-\beta \mu \bar{\rho}_{\mathrm{b}}$, where $\bar{\rho}_{\mathrm{b}}=a^{3} \rho$. The equilibrium condition, $\partial \Omega_{\mathrm{b}} / \partial \bar{\rho}_{\mathrm{b}}=0$, leads to a nonlinear equation, which we solved numerically. The solution reveals the existence of two phases, enriched in ions and solvent, which we call IL-rich and IL-poor phases. Figure 2a (solid black lines) shows that there is a region of temperatures and IL densities, where an IL-solvent mixture separates into the IL-poor and IL-rich phases. This region shrinks for increasing temperature and ends at a critical point $\bar{T}_{\mathrm{c}}=k_{\mathrm{B}} T_{\mathrm{c}} a^{3} / K \approx 0.09$ and $\rho_{\mathrm{c}} a^{3} \approx 0.25$. $^{36}$

The bulk phase diagram shown in Figure $2 \mathrm{a}$ is typical for IL-solvent mixtures. ${ }^{41,42}$ Various imidazolium tetrafluoroborate ILs in arenes, alkohols, and water have been reported to have critical temperatures in the range from 300 to $400 \mathrm{~K}$ and critical IL mole fractions from as low as 0.02 to about $0.125 .{ }^{42}$ A popular 1-hexyl-3-methylimidazolium tetrafluoroborate $\left(\mathrm{C} 6 \mathrm{mim}-\mathrm{BF}_{4}\right)$ has the critical temperature $T_{\mathrm{c}} \approx 326 \mathrm{~K}$ and critical mole fraction $x_{\mathrm{c}} \approx 0.125$ in alkohol $\left(\mathrm{C}_{6} \mathrm{OH}\right)$ and $T_{\mathrm{c}} \approx$ $331 \mathrm{~K}$ and $x_{\mathrm{c}} \approx 0.04$ in water. ${ }^{42}$ However, aqueous bistriflimide (TFSI)-based ILs have higher critical concentrations (between 0.2 and 0.3 ), closer to our model, with the critical temperature ranging from 400 to $420 \mathrm{~K}^{41}$

Capillary Ionization of Uncharged Pores. The bulk phase behavior of IL-solvent mixtures translates into a similar behavior in uncharged mesopores, where the phase separation region is shifted (symbols in Figure 2a). Examples of the inpore ion density profiles at coexistence are shown in Figure $2 b$. The phase diagram in the plane of temperature and inverse slit width consists of a line of first-order phase transitions between the IL-poor and IL-rich phases (Figure 2c). Thus, a phase transition can be induced by changing temperature or slit width. A transition as a function of temperature is illustrated in Figure $2 \mathrm{~d}$. This figure shows the amount of an IL adsorbed in the mesopore,

$$
\Gamma=\int_{0}^{w} \rho(z) \mathrm{d} z
$$

and demonstrates a capillary ionization obtained upon decreasing temperature; that is, that the amount of the IL adsorbed in the pore increases abruptly at the transition as the temperature is decreased.

The location of a capillary ionization transition can be estimated using the Kelvin equation, ${ }^{43,44}$ which in our case reads (Section S2)

$$
\mu_{c i}=\mu_{\text {bulk }}-\frac{2 \Delta \gamma}{w \Delta \rho}
$$

where $\mu_{\text {bulk }}$ is the chemical potential of the bulk transition, $\Delta \rho$ is the jump of the IL density at the transition, and $\Delta \gamma$ is the difference in the wall-fluid surface tensions of the IL-poor and IL-rich phases. To calculate the surface tensions, we used a semi-infinite system consisting of a single flat electrode. The prediction from eq 4 is shown by blue solid line in Figure 2a and demonstrates good quantitative agreement with the full numerical calculations (see also Figure S1).

Voltage-Induced Capillary lonization. The black line in Figure 3 a shows that the region of the IL-rich phase widens as 


\section{a}

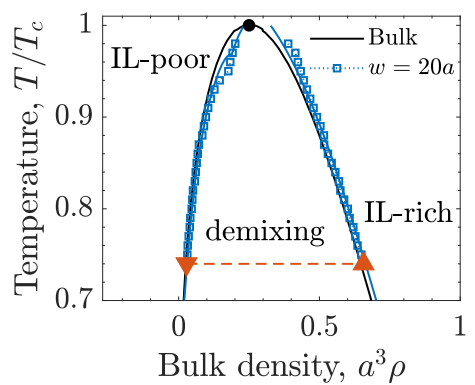

c

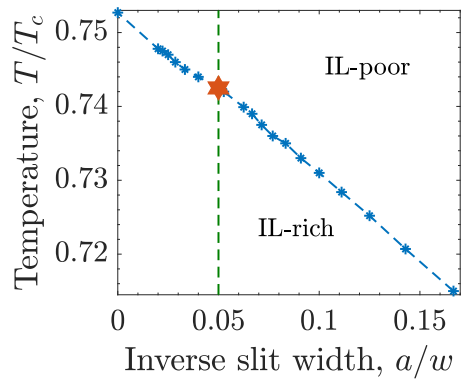

b

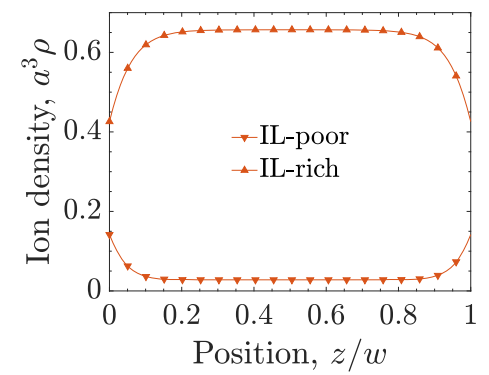

$\mathrm{d}$

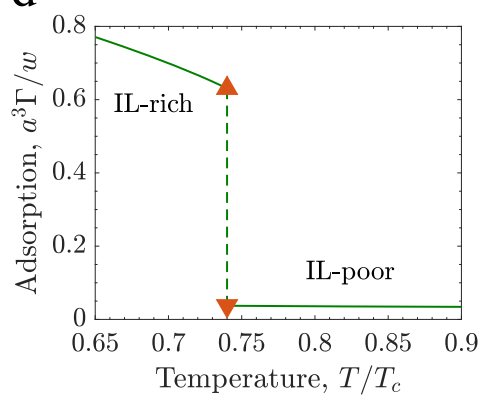

Figure 2. Capillary ionization of uncharged slit mesopores. (a) Phase diagram in the temperature-ion density plane. The solid black line shows the bulk diagram and the circle denotes the critical point. The open squares show the results for a slit of width $w=20 a$ obtained by numerically minimizing eq 1 , and the blue lines show the results obtained by the Kelvin equation, eq 4 . The triangles and the dashed line show the temperature $T / T_{\mathrm{c}}=0.74$ and the densities at coexistence obtained at the chemical potential $\mu / k_{\mathrm{B}} T_{\mathrm{c}}=-4.57$; this value of the chemical potential is used in the other panels. Phase diagrams in the chemical potential-temperature and ionophilicity-temperature planes are shown in Figures S1 and S2. (b) Inpore ion density profiles at the coexistence indicated in panel (a). (c) Diagram showing capillary phase transitions in the plane of temperature and inverse slit width. The vertical dash line indicates the slit width used in the other panels. (d) Amount of IL adsorbed in the pore, $\Gamma$, as a function of temperature for the slit width $w=20 a$. $\Gamma$ is given by eq 3 . In all plots the ionophilicity $a^{3} h_{\mathrm{s}} / \xi_{0}=0.25$, where $\xi_{0}$ is the bare correlation length and $a$ the ion diameter.

a voltage is applied to a pore with respect to the bulk electrolyte. The applied potential creates favorable conditions for the counterions to reside inside the pore, which bring along the co-ions (Figure 3e). At high voltages, this region shrinks. The difference between the ion structures near the pore walls in the IL-rich and IL-poor phases decreases for increasing voltage (Figures S6 and S7). Thus, the thermodynamic state becomes determined more and more by the in-pore bulk region, favoring the IL-poor phase at given thermodynamic conditions (Figure S1).

Outside of any transition, ion adsorption ( $\Gamma$, eq 3 ), accumulated charge,

$$
Q=\int_{0}^{w} c(z) \mathrm{d} z
$$

and the differential capacitance,

$$
C(U)=\frac{\mathrm{d} Q}{\mathrm{~d} U}
$$

are all continuous functions of voltage (the blue dashed lines in Figure $3 b-d)$. At low temperatures, the system is in the IL-rich state, characterized by a high ion density, and hence the capacitance has a bell shape $\left(T / T_{c}=0.72\right.$ in Figure $\left.3 d\right)$, in accord with numerous studies. ${ }^{45-53}$ At higher temperatures, the IL-poor phase becomes stable and the shape of the capacitance becomes bird-like $\left(T / T_{c}=0.78\right.$ and $T / T_{c}=0.838$ in Figure $3 \mathrm{~d}) .{ }^{36,54}$ The charging mechanisms in these two cases differ significantly, as demonstrated by the charging parame$\operatorname{ter}^{55,56}$

$$
X_{\mathrm{D}}(U)=\frac{e}{C(U)} \frac{\mathrm{d} \Gamma}{\mathrm{d} U}
$$

shown in Figure 3e. In the IL-poor phase at low voltages $\left(T / T_{c}\right.$ $=0.72$ and $T / T_{\mathrm{c}}=0.78$ in Figure $\left.3 \mathrm{e}\right), X_{\mathrm{D}}$ quickly becomes larger than unity, which means that charging proceeds by adsorption of both co-ions and counterions. In the IL-rich phase $\left(T / T_{c}=0.838\right.$ in Figure $\left.3 \mathrm{e}\right)$, we have $0<X_{\mathrm{D}}<1$ in the whole range of voltages and hence charging is a combination of counterion adsorption and co-ions swapping for counterions.

Applying a voltage to a mesopore can induce a capillary ionization transition (Figure 3a,b). This transition is accompanied by a sharp increase of the charge accumulated in the pore (Figure 3c), which has important consequences for capacitance and energy storage. To analyze charging in this case, we write for the accumulated charge

$$
Q(U)=Q_{\text {poor }}(U)+\left[Q_{\text {rich }}(U)-Q_{\text {poor }}(U)\right] \theta\left(U-U_{\text {ci }}\right)
$$

where $Q_{\text {rich }}(U)$ and $Q_{\text {poor }}(U)$ are the charge accumulated in the pore in the IL-rich and IL-poor phases, respectively, $U_{\mathrm{ci}}$ is the transition voltage, and $\theta(x)$ is the Heaviside step function, equal to unity for $x>0$ and zero otherwise. Correspondingly, the differential capacitance diverges at the transition, viz., $C\left(U_{\mathrm{ci}}\right)=C_{\text {poor }}\left(U_{\mathrm{ci}}\right)+\Delta Q_{\mathrm{ci}} \delta\left(U-U_{\mathrm{ci}}\right)$, where $\delta(x)$ is the Dirac delta function, $C_{\text {poor }}(U)=\mathrm{d} Q_{\text {poor }} / \mathrm{d} U$ is the capacitance in the IL-poor phase, and

$$
\Delta Q_{\mathrm{ci}}=Q_{\text {rich }}\left(U_{\mathrm{ci}}\right)-Q_{\text {poor }}\left(U_{\mathrm{ci}}\right)
$$


a

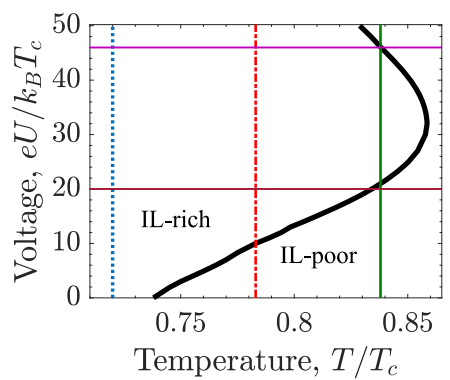

d

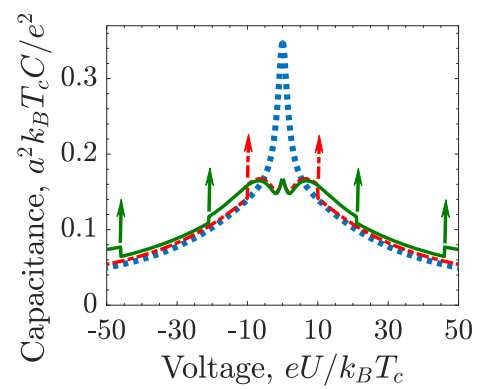

b

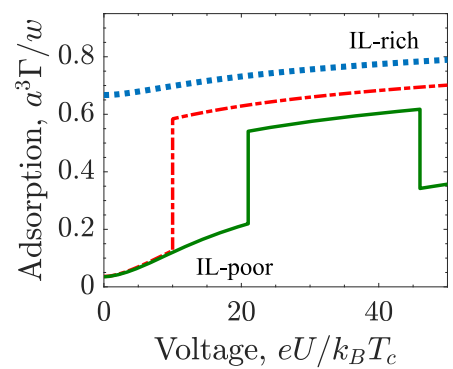

e

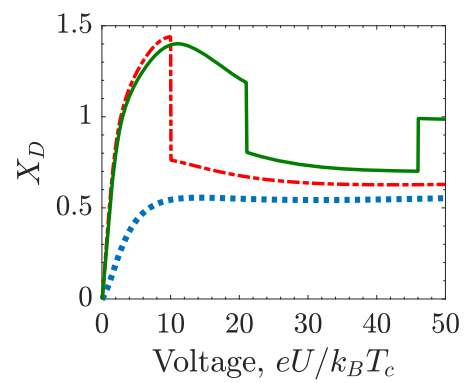

c

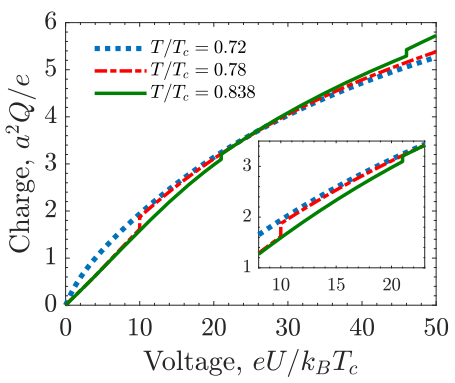

f

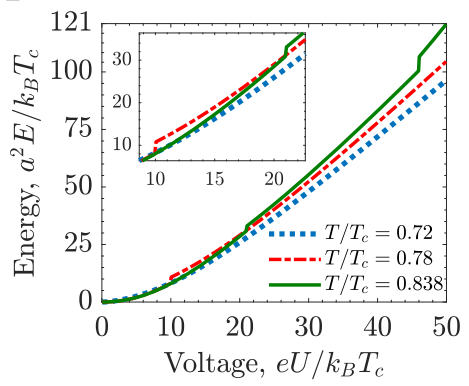

Figure 3. Voltage-induced capillary ionization and chargingof slit mesopores. (a) Phase diagram in the temperature-voltage plane. The thick black line shows a line of first-order transitions betweenthe IL-rich and IL-poor phases. The thin vertical lines indicate temperatures used inthe other panels. The horizontal lines show the values of voltage used inFigure 4. (b) Amount of IL adsorbed in the pore (eq 3), (c) charge accumulated in the pore (eq 5), (d) differential capacitance (eq 6), (e) charging parameter $X_{\mathrm{D}}$ (eq 1) and (f) energy stored inthe pore (eq 4) as functions of the applied voltage for three values of temperature indicated in (a). The capacitance for $T / T_{\mathrm{c}}=0.78$ and $T / T_{\mathrm{c}}=0.838$ diverges at the transitions, as schematically denoted by upward pointing arrows. In all plots the pore width $w=20 a$, chemical potential $\mu / k_{\mathrm{B}} T_{\mathrm{c}}=-4.57$ and ionophilicity $a^{3} h_{\mathrm{s}} / \xi_{0}$ $=0.25$, where $\xi_{0}$ is the bare correlation length and $a$ isthe ion diameter.For typical values of the ion diameter $a=0.7 \mathrm{~nm}$ and room temperature for $T_{\mathcal{c}}$, the various units are thermal voltage $e / k_{\mathrm{B}} T_{\mathrm{c}} \approx 26 \mathrm{mV}$ for voltage, $e / a^{2} \approx 2 e \mathrm{~nm}^{-2} \approx 32 \mu \mathrm{C} \mathrm{cm}^{-2}$ for accumulated charge, thermal electric capacitance $e^{2} /\left(k_{\mathrm{B}} T_{\mathrm{c}} a^{2}\right) \approx 620 \mu \mathrm{F} \mathrm{cm}{ }^{-2}$ for capacitance, and $k_{\mathrm{B}} T_{\mathrm{c}} / a^{2} \approx 0.84 \mathrm{~mJ} \mathrm{~cm}^{-2} \approx 0.23 \mathrm{nW} \mathrm{cm} \mathrm{cor}^{-2}$ forergy. The color and line codes are the same in panels (b)-(f). Phase diagrams in the chemical potential-temperature plane for a few voltages are shown in Figure S3.. The diagrams for a few other values of the chemical potential are presented in Figure S4 and for a few values of the Bjerrum length in Figure S5. Examples of the total and charge density profiles at the coexistence are shown in Figures S6 and S7.

is the jump of the accumulated charge at the transition voltage $U_{\text {ci. }}$ Thus, the energy stored in the pore,

$$
E(U)=\int_{0}^{U} C(v) v \mathrm{~d} v
$$

acquires an additional contribution at the transition, $\Delta E_{\mathrm{ci}}=$ $U_{\mathrm{ci}} \Delta Q_{\mathrm{ci}}$, which appears as a jump in the stored energy (Figure 3f).

Of course, the jumps of the accumulated charge obtained upon voltage increase are always positive (Figure 3c). Because of the re-entrant behavior, therefore, a jump $\Delta Q_{\mathrm{ci}}$ from the ILpoor to the IL-rich phase (eq 9) is positive for $U<U_{\mathrm{b}}$ and negative for $U>U_{\mathrm{b}}$, where $U_{\mathrm{b}} \approx 32 k_{\mathrm{B}} T_{\mathrm{c}} / e$ is the voltage at which the transition line bends (Figure 3a). In Figure 4a,b, we show examples of the accumulated charge and stored energy density as functions of temperature for $U<U_{\mathrm{b}}$ and $U>U_{\mathrm{b}}$. It is worth noting that there is no monotonous relation between the accumulated charge $Q$ (or integral capacitance $C_{I}=Q / U$ ) and the stored energy density $E$, as one might expect from the $E=C_{\mathrm{I}} U^{2} / 2$ expression. In particular, an increase in the stored energy may accompany a decrease (Figure $4 \mathrm{a}$ ) or an increase (Figure $4 \mathrm{~b}$ ) in the accumulated charge. Clearly, the $C_{\mathrm{I}} U^{2} / 2$ equation is only valid in the linear regime $(Q \sim U)$, while the actual charge-voltage relation is more complex.

In Figure $4 \mathrm{c}$, we summarize this behavior by plotting $\Delta Q_{\mathrm{ci}}$ and $\Delta E_{\mathrm{ci}}=U_{\mathrm{ci}} \Delta Q_{\mathrm{ci}}$ along the transition line of Figure $3 \mathrm{a}$.
Remarkably, the magnitudes of $\Delta Q_{\mathrm{ci}}$ and $\Delta E_{\mathrm{ci}}$ increase steeply with voltage at high voltages.

\section{CONCLUSION}

We have studied ionic liquid-solvent mixtures in slit mesopores. We revealed that a pore could become spontaneously ionized as a function of temperature, slit width, or applied voltage, as manifested by jumps in the amount of an ionic liquid adsorbed in the pores. Voltageinduced capillary ionization transitions are exciting as they can be re-entrant and create jumps in the accumulated charge and stored energy density. The possibility to obtain a sharp increase in the stored energy by minute changes of voltage or temperature is spectacular and may find useful technological applications.

Our predictions are based on a simple mean-field model and ideal slit mesopores. In real systems, the locations of the transitions might be shifted by fluctuations, high-density steric repulsions, ${ }^{57}$ and chemical complexity of ion-solvent interactions, all neglected in this work. Furthermore, the distribution of pore sizes and shapes in real electrodes may smear out the jumps at the transitions. ${ }^{14,58,59}$ However, the rapid progress in low-dimensional carbon materials provides hope for engineering highly monodisperse porous structures, e.g., based on MXene ${ }^{60,61}$ or graphene, ${ }^{62-64}$ which would facilitate experimental observation of capillary ionization 
a
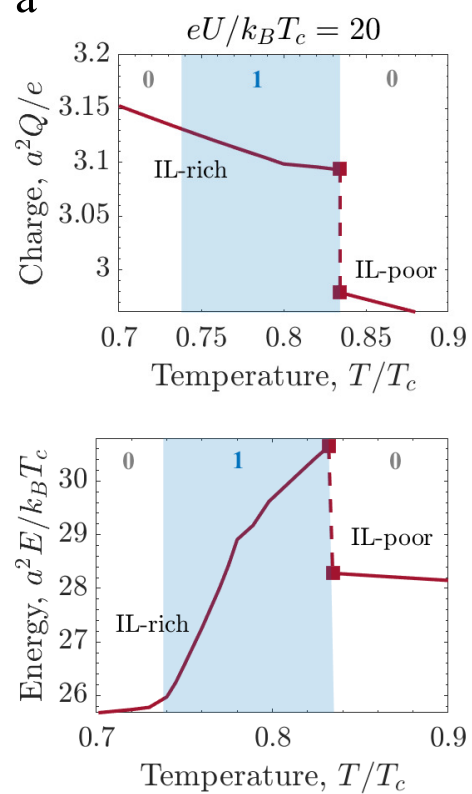

b
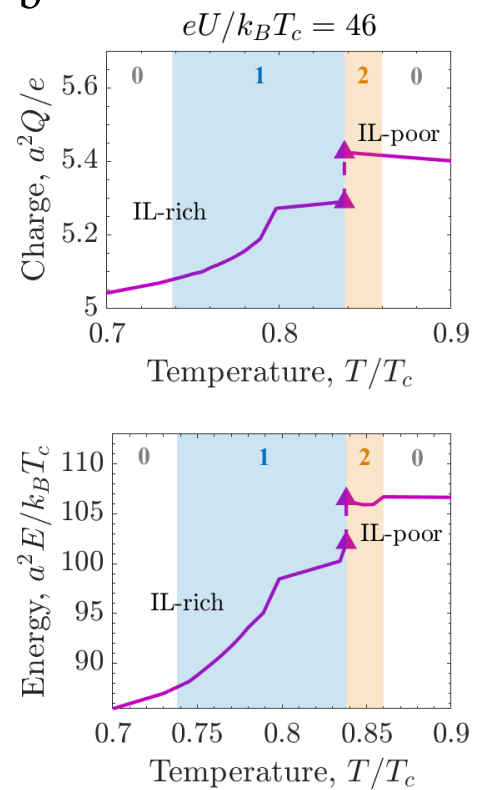

C
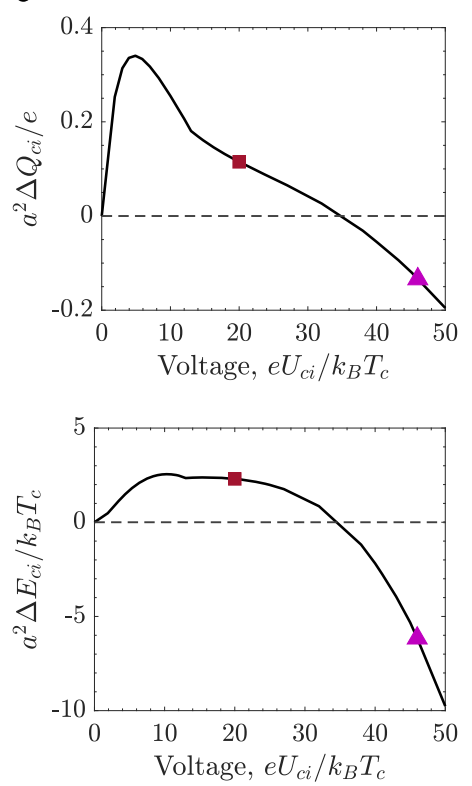

Figure 4. Charge and energy storage in slit mesopores. (a) Accumulated charge (top) and stored energy (bottom) as functions of temperature for appliedvoltage $e U / k_{\mathrm{B}} T_{\mathrm{c}}=20$. (b) Same as in (a) but for $e U / k_{\mathrm{B}} T_{\mathrm{c}}=46$. The shaded areas show the regions with one and two transitions occurring at voltages below those indicated onthe plots. The number of transitions in each region is also indicated on the plots. (c) Jumps in the accumulated charge (top) and stored energy (bottom) along the transition line as functions of the transition voltage $U_{\mathrm{ci}}$. The symbols correspond to the jumps shown in (a) and (b). Chemical potential $\mu / k_{\mathrm{B}} T_{\mathrm{c}}=-4.57$, slit width $w=20 a$, and ionophilicity $a^{3} h_{\mathrm{s}} / \xi_{0}=0.25$, where $\xi_{0}$ is the bare correlation length and $a$ the ion diameter. For typical values of the ion diameter $a=0.7 \mathrm{~nm}$ and room temperature for $T_{\mathcal{c}}$, the various units are thermal voltage $e / k_{\mathrm{B}} T_{\mathrm{c}} \approx 26 \mathrm{mV}$ for voltage, $e / a^{2} \approx 2 e \mathrm{~nm}^{-2} \approx 32 \mu \mathrm{C} \mathrm{cm}^{-2}$ for accumulated charge, and $k_{\mathrm{B}} T_{\mathrm{c}} / a^{2} \approx 0.84 \mathrm{~mJ} \mathrm{~cm}^{-2} \approx 0.23 \mathrm{nW} \mathrm{cm}{ }^{-2}$ for energy. For the phase diagram, seeFigure 3 a.

transitions and enable their application in heat-to-electricity conversion and energy storage.

\section{ASSOCIATED CONTENT}

\section{(s) Supporting Information}

The Supporting Information is available free of charge at https://pubs.acs.org/doi/10.1021/acs.jpcc.1c00624.

Details of the numerical approach, the Kelvin equation, supplementary plots of phase diagrams and of ion and charge densities (PDF)

\section{AUTHOR INFORMATION}

\section{Corresponding Author}

Svyatoslav Kondrat - Institute of Physical Chemistry, Polish Academy of Sciences, 01-224 Warsaw, Poland; Max-PlanckInstitut für Intelligente Systeme, D-70569 Stuttgart, Germany; IV. Institut für Theoretische Physik, Universität Stuttgart, D-70569 Stuttgart, Germany; (1) orcid.org/00000003-4448-0686; Email: svyatoslav.kondrat@gmail.com, skondrat@ichf.edu.pl

\section{Authors}

Carolina Cruz - Institute of Physical Chemistry, Polish Academy of Sciences, 01-224 Warsaw, Poland; (1) orcid.org/ 0000-0002-6319-0740

Enrique Lomba - Instituto de Química Física Rocasolano, CSIC, E-28006 Madrid, Spain; ㅇ orcid.org/0000-0002$4768-2040$

Alina Ciach - Institute of Physical Chemistry, Polish Academy of Sciences, 01-224 Warsaw, Poland; (1) orcid.org/00000002-5556-401X
Complete contact information is available at:

https://pubs.acs.org/10.1021/acs.jpcc.1c00624

\section{Notes}

The authors declare no competing financial interest.

\section{ACKNOWLEDGMENTS}

We thank Mathijs Janssen (University of Oslo) for critical reading of the manuscript and insightful comments and suggestions. This work received funding from the European Unions Horizon 2020 research and innovation programmes under the Marie Skłodowska-Curie grant agreement No. 711859 to C.C. and No. 734276 to A.C. and S.K. Additional funding was received from the Ministry of Science and Higher Education of Poland for the implementation of the international cofinanced projects No. 734276 in the years 2017-2021 and No. 711859 in the years 2017-2022.

\section{REFERENCES}

(1) Miller, J. R.; Simon, P. Materials Science Electrochemical Capacitors for Energy Management. Science 2008, 321, 651-652.

(2) Simon, P.; Gogotsi, Y. Materials for electrochemical capacitors. Nat. Mater. 2008, 7, 845-854.

(3) Béguin, F.; Presser, V.; Balducci, A.; Frackowiak, E. Carbons and Electrolytes for Advanced Supercapacitors. Adv. Mater. 2014, 26, 2219-2251.

(4) González, A.; Goikolea, E.; Barrena, J. A.; Mysyk, R. Review on supercapacitors: Technologies and materials. Renewable Sustainable Energy Rev. 2016, 58, 1189-1206.

(5) Porada, S.; Zhao, R.; van der Wal, A.; Presser, V.; Biesheuvel, P. M. Review on the science and technology of water desalination by capacitive deionization. Prog. Mater. Sci. 2013, 58, 1388-1442. 
(6) Suss, M. E.; Presser, V. Water Desalination with Energy Storage Electrode Materials. Joule 2018, 2, 10-15.

(7) Zhang, Y.; Srimuk, P.; Aslan, M.; Gallei, M.; Presser, V. Polymer ion-exchange membranes for capacitive deionization of aqueous media with low and high salt concentration. Desalination 2020, 479, 114331.

(8) Brogioli, D. Extracting Renewable Energy from a Salinity Difference Using a Capacitor. Phys. Rev. Lett. 2009, 103, 058501.

(9) Härtel, A.; Janssen, M.; Weingarth, D.; Presser, V.; van Roij, R. Heat-to-current conversion of low-grade heat from a thermocapacitive cycle by supercapacitors. Energy Environ. Sci. 2015, 8, 2396-2401.

(10) Janssen, M.; van Roij, R. Reversible Heating in Electric Double Layer Capacitors. Phys. Rev. Lett. 2017, 118, 096001.

(11) Chmiola, J.; Yushin, G.; Gogotsi, Y.; Portet, C.; Simon, P.; Taberna, P. L. Anomalous Increase in Carbon Capacitance at Pore Sizes Less Than 1 Nanometer. Science 2006, 313, 1760.

(12) Raymundo-Piñero, E.; Kierczek, K.; Machnikowski, J.; Béguin, F. Relationship between the nanoporous texture of activated carbons and their capacitance properties in different electrolytes. Carbon 2006, 44, 2498-2507.

(13) Kondrat, S.; Kornyshev, A. Superionic state in double-layer capacitors with nanoporous electrodes. J. Phys.: Condens. Matter 2011, 23, 022201 .

(14) Kondrat, S.; Pérez, C. R.; Presser, V.; Gogotsi, Y.; Kornyshev, A. A. Effect of pore size and its dispersity on the energy storage in nanoporous supercapacitors. Energy Environ. Sci. 2012, 5, 6474.

(15) Péan, C.; Merlet, C.; Rotenberg, B.; Madden, P. A.; Taberna, P.-L.; Daffos, B.; Salanne, M.; Simon, P. On the Dynamics of Charging in Nanoporous Carbon-Based Supercapacitors. ACS Nano 2014, 8, 1576.

(16) Pak, A. J.; Hwang, G. S. Charging Rate Dependence of Ion Migration and Stagnation in Ionic-Liquid-Filled Carbon Nanopores. J. Phys. Chem. C 2016, 120, 24560.

(17) Breitsprecher, K.; Holm, C.; Kondrat, S. Charge Me Slowly, I Am in a Hurry: Optimizing Charge-Discharge Cycles in Nanoporous Supercapacitors. ACS Nano 2018, 12, 9733-9741.

(18) Breitsprecher, K.; Janssen, M.; Srimuk, P.; Mehdi, B. L.; Presser, V.; Holm, C.; Kondrat, S. How to speed up ion transport in nanopores. Nat. Commun. 2020, 11, 6085.

(19) Vasilyev, O. A.; Kornyshev, A. A.; Kondrat, S. Connections Matter: On the Importance of Pore Percolation for Nanoporous Supercapacitors. ACS Appl. Energy Mater. 2019, 2, 5386-5390.

(20) Mysyk, R.; Raymundo-Piñero, E.; Béguin, F. Saturation of subnanometer pores in an electric double-layer capacitor. Electrochem. Commun. 2009, 11, 554-556.

(21) Hesse, S. A.; Fritz, K. E.; Beaucage, P. A.; Thedford, R. P.; Yu, F.; DiSalvo, F. J.; Suntivich, J.; Wiesner, U. Materials Combining Asymmetric Pore Structures with Well-Defined Mesoporosity for Energy Storage and Conversion. ACS Nano 2020, 14, 16897-16906.

(22) Ong, S. P.; Andreussi, O.; Wu, Y.; Marzari, N.; Ceder, G. Electrochemical Windows of Room-Temperature Ionic Liquids from Molecular Dynamics and Density Functional Theory Calculations. Chem. Mater. 2011, 23, 2979-2986.

(23) Hayyan, M.; Mjalli, F. S.; Hashim, M. A.; AlNashef, I. M.; Mei, $\mathrm{T}$. X. Investigating the electrochemical windows of ionic liquids. J. Ind. Eng. Chem. 2013, 19, 106-112.

(24) Kazemiabnavi, S.; Zhang, Z.; Thornton, K.; Banerjee, S. Electrochemical Stability Window of Imidazolium-Based Ionic Liquids as Electrolytes for Lithium Batteries. J. Phys. Chem. B 2016, 120, 5691-5702.

(25) Lian, C.; Liu, H.; Li, C.; Wu, J. Hunting ionic liquids with large electrochemical potential windows. AIChE J. 2019, 65, 804-810.

(26) Tokuda, H.; Baek, S.-J.; Watanabe, M. Room-Temperature Ionic Liquid-Organic Solvent Mixtures: Conductivity and Ionic Association. Electrochemistry 2005, 73, 620-622.

(27) Chaban, V. V.; Voroshylova, I. V.; Kalugin, O. N.; Prezhdo, O. V. Acetonitrile Boosts Conductivity of Imidazolium Ionic Liquids. J. Phys. Chem. B 2012, 116, 7719-7727.
(28) Rilo, E.; Vila, J.; García-Garabal, S.; Varela, L. M.; Cabeza, O. Electrical Conductivity of Seven Binary Systems Containing 1-Ethyl3-methyl Imidazolium Alkyl Sulfate Ionic Liquids with Water or Ethanol at Four Temperatures. J. Phys. Chem. B 2013, 117, 14111418.

(29) Montes-Campos, H.; Kondrat, S.; Rilo, E.; Cabeza, O.; Varela, L. M. Random-Alloy Model for the Conductivity of Ionic LiquidSolvent Mixtures. J. Phys. Chem. C 2020, 124, 11754-11759.

(30) Burt, R.; Breitsprecher, K.; Daffos, B.; Taberna, P.-L.; Simon, P.; Birkett, G.; Zhao, X. S.; Holm, C.; Salanne, M. Capacitance of Nanoporous Carbon-Based Supercapacitors Is a Trade-Off between the Concentration and the Separability of the Ions. J. Phys. Chem. Lett. 2016, 7, 4015.

(31) Evans, R.; Marconi, U. M. B.; Tarazona, P. Fluids in narrow pores: Adsorption, capillary condensation, and critical points. J. Chem. Phys. 1986, 84, 2376-2399.

(32) Evans, R. Fluids adsorbed in narrow pores: phase equilibria and structure. J. Phys.: Condens. Matter 1990, 2, 8989-9007.

(33) Jagiello, J.; Thommes, M. Comparison of DFT characterization methods based on $\mathrm{N} 2, \mathrm{Ar}, \mathrm{CO} 2$, and $\mathrm{H} 2$ adsorption applied to carbons with various pore size distributions. Carbon 2004, 42, $1227-$ 1232.

(34) Ravikovitch, P. I.; Neimark, A. V. Density Functional Theory Model of Adsorption on Amorphous and Microporous Silica Materials. Langmuir 2006, 22, 11171-11179.

(35) Kupgan, G.; Liyana-Arachchi, T. P.; Colina, C. M. NLDFT Pore Size Distribution in Amorphous Microporous Materials. Langmuir 2017, 33, 11138-11145.

(36) Cruz, C.; Ciach, A.; Lomba, E.; Kondrat, S. Electrical Double Layers Close to Ionic Liquid-Solvent Demixing. J. Phys. Chem. C 2019, 123, 1596-1601.

(37) Cruz, C.; Kondrat, S.; Lomba, E.; Ciach, A. Effect of proximity to ionic liquid-solvent demixing on electrical double layers. J. Mol. Liq. 2019, 294, 111368 .

(38) Catenaccio, A.; Daruich, Y.; Magallanes, C. Temperature dependence of the permittivity of water. Chem. Phys. Lett. 2003, 367, 669-671.

(39) Gagliardi, L. G.; Castells, C. B.; Ràfols, C.; Rosés, M.; Bosch, E. Static Dielectric Constants of Acetonitrile/Water Mixtures at Different Temperatures and Debye-Hückel $A$ and $a_{0} B$ Parameters for Activity Coefficients. J. Chem. Eng. Data 2007, 52, 1103-1107.

(40) Riniker, S.; Horta, B. A. C.; Thijssen, B.; Gupta, S.; van Gunsteren, W. F.; Hünenberger, P. H. Temperature Dependence of the Dielectric Permittivity of Acetic Acid, Propionic Acid and Their Methyl Esters: A Molecular Dynamics Simulation Study. ChemPhysChem 2012, 13, 1182-1190.

(41) Rotrekl, J.; Storch, J.; Velísek, P.; Schröer, W.; Jacquemin, J.; Wagner, Z.; Husson, P.; Bendová, M. Liquid Phase Behavior in Systems of 1-Butyl-3-alkylimidazolium bis\{(trifluoromethyl)sulfonyl\}imide Ionic Liquids with Water: Influence of the Structure of the C5 Alkyl Substituent. J. Solution Chem. 2017, 46, 1456-1474.

(42) Butka, A.; Vale, V. R.; Saracsan, D.; Rybarsch, C.; Weiss, V. C.; Schröer, W. Liquid-liquid phase transition in solutions of ionic liquids with halide anions: Criticality and corresponding states. Pure Appl. Chem. 2008, 80, 1613-1630.

(43) Thomson, W. 4. On the Equilibrium of Vapour at a Curved Surface of Liquid. Proc. R. Soc. Edinburgh 1872, 7, 63-68.

(44) Fisher, L. R.; Gamble, R. A.; Middlehurst, J. The Kelvin equation and the capillary condensation of water. Nature 1981, 290, $575-576$.

(45) Kornyshev, A. A. Double-Layer in Ionic Liquids: Paradigm Change? J. Phys. Chem. B 2007, 111, 5545-5557.

(46) Alam, M.; Islam, M.; Okajima, T.; Ohsaka, T. Measurements of differential capacitance in room temperature ionic liquid at mercury, glassy carbon and gold electrode. Electrochem. Commun. 2007, 9, 2370-2374.

(47) Islam, M. M.; Alam, M. T.; Ohsaka, T. Electrical Double-Layer Structure in Ionic Liquids: A Corroboration of the Theoretical Model by Experimental Results. J. Phys. Chem. C 2008, 112, 16568-16574. 
(48) Lockett, V.; Sedev, R.; Ralston, J.; Horne, M.; Rodopoulos, T. Differential Capacitance of the Electrical Double Layer in Imidazolium-Based Ionic Liquids: Influence of Potential, Cation Size, and Temperature. J. Phys. Chem. C 2008, 112, 7486-7495.

(49) Lamperski, S.; Outhwaite, C. W.; Bhuiyan, L. B. The Electric Double-Layer Differential Capacitance at and near Zero Surface Charge for a Restricted Primitive Model Electrolyte. J. Phys. Chem. B 2009, 113, 8925-8929.

(50) Klos, J.; Lamperski, S. Electrical Double Layer Properties in Diameter Asymmetric Molten Salt Investigated by Grand Canonical Monte Carlo Method. J. Phys. Chem. C 2010, 114, 13329-13333.

(51) Henderson, D.; Lamperski, S.; Bhuiyan, L. B.; Wu, J. The tail effect on the shape of an electrical double layer differential capacitance curve. J. Chem. Phys. 2013, 138, 144704.

(52) Bossa, G. V.; Caetano, D. L. Z.; de Carvalho, S. J.; Bohinc, K.; May, S. Modeling the camel-to-bell shape transition of the differential capacitance using mean-field theory and Monte Carlo simulations. Eur. Phys. J. E: Soft Matter Biol. Phys. 2018, 41, 113.

(53) Islam, M. M.; Ohsaka, T. Model of electrical double layer structure at semi-metallic electrode/ionic liquid interface. Electrochim. Acta 2021, 368, 137555.

(54) Alam, M. T.; Islam, M. M.; Okajima, T.; Ohsaka, T. Capacitance Measurements in a Series of Room-Temperature Ionic Liquids at Glassy Carbon and Gold Electrode Interfaces. J. Phys. Chem. C 2008, 112, 16600-16608.

(55) Forse, A. C.; Merlet, C.; Griffin, J. M.; Grey, C. P. New Perspectives on the Charging Mechanisms of Supercapacitors. J. Am. Chem. Soc. 2016, 138, 5731-5744.

(56) Breitsprecher, K.; Abele, M.; Kondrat, S.; Holm, C. The effect of finite pore length on ion structure and charging. J. Chem. Phys. 2017, 147, 104708.

(57) Antypov, D.; Barbosa, M. C.; Holm, C. Incorporation of excluded-volume correlations into Poisson-Boltzmann theory. Phys. Rev. E 2005, 71, 061106.

(58) Kornyshev, A. A. Faraday Discuss. 2013, 164, 117-133.

(59) Kondrat, S.; Kornyshev, A. Pressing a spring: What does it take to maximize the energy storage in nanoporous supercapacitors? Nanoscale Horiz 2016, 1, 45-52.

(60) Naguib, M.; Mochalin, V. N.; Barsoum, M. W.; Gogotsi, Y. 25th Anniversary Article: MXenes: A New Family of Two-Dimensional Materials. Adv. Mater. 2014, 26, 992.

(61) Zhao, M.-Q.; Ren, C. E.; Ling, Z.; Lukatskaya, M. R.; Zhang, C.; Aken, K. L. V.; Barsoum, M. W.; Gogotsi, Y. Flexible MXene/ Carbon Nanotube Composite Paper with High Volumetric Capacitance. Adv. Mater. 2015, 27, 339-345.

(62) Yoo, J. J.; Balakrishnan, K.; Huang, J. S.; Meunier, V.; Sumpter, B. G.; Srivastava, A.; Conway, M.; Reddy, A. L. M.; Yu, J.; Vajtai, R.; et al. Ultrathin Planar Graphene Supercapacitors. Nano Lett. 2011, 11, $1423-1427$.

(63) Chen, J.; Li, C.; Shi, G. Graphene Materials for Electrochemical Capacitors. J. Phys. Chem. Lett. 2013, 4, 1244-1253.

(64) Yang, X.; Cheng, C.; Wang, Y.; Qiu, L.; Li, D. Liquid-Mediated Dense Integration of Graphene Materials for Compact Capacitive Energy Storage. Science 2013, 341, 534-537. 